\title{
Search for Odderon in the Parity Violating Amplitudes' Asymptotics
}

\section{Leszek Łukaszuk ${ }^{* \dagger}$}

Andrzej Soltan Institute for Nuclear Studies, Hoza 69, 00-681 Warsaw, Poland

E-mail: lukaszuk@fuw.edu.pl

Model dependent arguments are given for the possibility of Odderon-type asymptotics in the case of Parity Violating Compton Amplitude. Estimated contributions come from semi-hard region and are not related to the QCD Odderon.

DIFFRACTION 2006 - International Workshop on Diffraction in High-Energy Physics September 5-10 2006

Adamantas, Milos island, Greece

* Speaker.

${ }^{\dagger}$ This work was partially supported by Polish-French Scientific Agreement Polonium 


\section{Introduction}

We shall be concerned with maximal Odderon asymptotics, i.e. the situation when $\Delta \sigma^{\text {tot }} \rightarrow \infty$ as $\ln s$ at asymptotically large $s$, where $s$ denotes the center-of-mass energy squared and $\Delta \sigma^{\text {tot }}$ is the difference between the total cross sections for the given amplitude and its crossing counterpart. Odderon asymptotics emerged for the first time in Ref. [1] and is nowadays usually associated with $C=-1$ exchange in $t$-channel. Although the studies in QCD [2, 3] (see Refs. [4, 5] for further references) support existence of $C=-1$ exchange (QCD Odderon), it should be stressed that such exchange need not be mandatory for the Odderon type behavior. In fact I am going to discuss in this talk example of such a situation in the case of spin dependent amplitude. Let us remark here that for spin averaged amplitudes the gluon and sea distributions do not change under crossing. To avoid cancellation (up to unimportant valence quarks) at high energies one needs $C=-1$ exchange, QCD Odderon. It need not be so for spin dependent cases. Here under crossing the spin components change, so the difference of polarized gluon and sea contributions can enter the expression for $\Delta \sigma^{t o t}$.

\section{Amplitude and kinematical region considered}

We shall consider parity violating crossing - odd Compton amplitude $\Delta A$ :

$$
\Delta A=A(\gamma \uparrow, p)-A(\gamma \downarrow, p),
$$

where $p$ denotes the unpolarized proton and $\uparrow, \downarrow$ denote (real) photon helicities. The choice of amplitude (2.1) was dictated by its simplicity. Moreover, we have an interesting dichotomy: either $\Delta A$ has Odderon (not necessarily maximal) behavior at high energies,

$$
\frac{\Delta A}{z} \rightarrow c(z),
$$

with $c(z) \ln (z)$ non vanishing for asymptotically large $z$, or parity-violating analogue of GerasimovDrell-Hearn sum rule is satisfied [6],

$$
\int_{\omega_{t h}}^{\infty} \frac{\Delta \sigma^{t o t}}{\omega} d \omega=0
$$

where $\omega$ in (2.3) denotes laboratory energy.

In further considerations we shall stay in perturbative region for parton-parton cross sections (masses are neglected):

$$
\lambda \hat{s} \leq|\hat{t}| \leq(1-\lambda) \hat{s}
$$

where

$$
0<\lambda_{0} \leq \lambda<\frac{1}{2}
$$

and

$$
Q^{2}=\frac{4 \hat{t} \hat{u}}{\hat{s}}=4 \lambda(1-\lambda) \hat{s}
$$

with

$$
\ln \frac{Q^{2}}{\Lambda_{Q C D}^{2}} \gg 1,
$$


i.e.

$$
\ln \frac{\hat{s}}{\Lambda_{Q C D}^{2}}+\ln \left(4 \lambda_{0}\left(1-\lambda_{0}\right)\right) \gg 1 .
$$

(Let us mention that we might use other possible symmetric choice:

$$
Q^{2}=\frac{\hat{s} \hat{t} \hat{u}}{\hat{s}^{2}+\hat{t}^{2}+\hat{u}^{2}}=\hat{s} \frac{\lambda(1-\lambda)}{1-2 \lambda+2 \lambda^{2}},
$$

without essential change of our results.)

The parton-parton cross sections in the perturbative region (2.8) are small, but the large contributions to $\gamma$ proton total cross sections may be caused by large parton structure functions in the semi-hard regime. Therefore in what follows we shall demand that condition (2.8) should be satisfied together with $\hat{s} / s \ll 1$. Parton - parton cross sections will be taken in the lowest perturbative order, $\left(\alpha \alpha_{s}\right)$, while structure functions will be treated in the framework of model assumptions such as saturation mechanism [7] with scaling [8, 9, 10, 11].

\section{Flavor contributions to $\Delta \sigma^{t o t}(\gamma p)$}

We aim at estimate of $\Delta \sigma^{t o t}(\gamma p)$ defined as

$$
\Delta \sigma^{t o t}(\gamma p)=\sigma_{t o t}(\gamma \uparrow p)-\sigma_{t o t}(\gamma \downarrow p) .
$$

In the lowest order both $g-g$ and $g-q(\bar{q})$ initiated processes do not contribute to $\Delta \sigma^{\text {fot }}(\gamma p)$. For given flavor distributions $q^{(\gamma)}$ and $q^{N}$ we should obtain combination of spin dependent convolutions of cross sections with distributions of suitable initial distributions such as $q^{\gamma)} \otimes q^{N}, \bar{q}^{(\gamma)} \otimes q^{N}, q^{(\gamma)} \otimes$ $\bar{q}^{N}, \bar{q}^{(\gamma)} \otimes \bar{q}^{N}$. Denoting contribution coming from these flavors as $\Delta \sigma\left[q^{(\gamma)}, q^{N}\right]$ we get:

$$
\Delta \sigma\left[q^{(\gamma)}, q^{N}\right]=\iiint \delta q^{\gamma}\left(Q^{2}, x_{1}\right) q_{V}^{N}\left(Q^{2}, x_{2}\right) \frac{d \hat{\sigma}_{q^{\gamma} q^{N}}}{d \hat{t}} d \hat{t} d x_{1} d x_{2}
$$

where

$$
\begin{gathered}
\delta q^{\gamma}=q_{\uparrow}^{\gamma}-q_{\downarrow}^{\gamma}, \\
q_{\uparrow}^{\gamma}=\bar{q}_{\uparrow}^{\gamma},
\end{gathered}
$$

with

$$
q_{V}^{N}=q_{\uparrow}^{N}+q_{\downarrow}^{N}-\left(\bar{q}_{\uparrow}^{N}+\bar{q}_{\downarrow}^{N}\right)
$$

and

$$
\frac{d \hat{\sigma}_{q^{\gamma} q^{N}}}{d \hat{t}}=\frac{d \hat{\sigma}\left(q_{\uparrow}^{\gamma}, q_{\uparrow}^{N}\right)}{d \hat{t}}-\frac{d \hat{\sigma}\left(q_{\downarrow}^{\gamma}, q_{\downarrow}^{N}\right)}{d \hat{t}}+\frac{d \hat{\sigma}\left(\bar{q}_{\uparrow}^{\gamma}, q_{\downarrow}^{N}\right)}{d \hat{t}}-\frac{d \hat{\sigma}\left(\bar{q}_{\downarrow}^{\gamma}, q_{\uparrow}^{N}\right)}{d \hat{t}} .
$$

Considerable simplification of expressions appearing in (3.2)-(3.6) follows from CP invariance together with the symmetrical form of $Q^{2}$ (see (2.6):

$$
Q^{2}(\hat{t}, \hat{u})=Q^{2}(\hat{u}, \hat{t})
$$




\section{Scaling and saturation}

Resummation of leading contributions in semi-hard region to polarized gluon and sea quark distributions indicates [12] violation of unitarity at small $x$ in analogy with spin averaged case. (Notice however that [13] come to different conclusions - we shall not follow such possibility in this note.) Therefore, using results of [12] we shall use - by analogy with unpolarized case - hypothesis of saturation and scaling $[7,8,9,10,11,14,15]$. In what follows we shall use sea quark distributions for real photon following analogous definitions for unpolarized distributions for nucleon (compare with Refs [7, 10, 14, 15]):

$$
\begin{gathered}
\delta q^{\gamma}=\alpha\left(\frac{e_{q}}{e}\right)^{2} F\left(x, Q^{2}\right) \frac{1}{x}, \\
\tau=\frac{Q^{2}}{Q_{s}^{2}}(x) \\
Q_{s}^{2}(x)=Q_{0}^{2}\left(\frac{x}{x_{0}}\right)^{-\Delta} .
\end{gathered}
$$

In the saturation region, i.e. $\tau \leq 1$, one has

$$
F=F_{\text {sat }}=D\left(\alpha_{s}\right) \frac{1}{\alpha_{s}} Q^{2} R_{h}^{2},
$$

$\alpha_{s} \leq D \leq 1$. In what follows we shall use fixed coupling $\alpha_{s}$. For $\tau>1$ we use

$$
F=F_{\text {scal }}=\frac{1}{\tau} F_{\text {sat }} .
$$

\section{Results}

We shall estimate contributions of given flavors $q^{\gamma}, q^{N}$ (3.2) to $\Delta \sigma^{t o t}$,

$$
\Delta \sigma\left(q^{(\gamma)}, q^{N}\right)=\iiint \delta q^{\gamma}\left(Q^{2}, x_{1}\right) q_{V}^{N}\left(Q^{2}, x_{2}\right) \frac{d \hat{\sigma}_{q^{\gamma} q^{N}}}{d \hat{t}} d \hat{t} d x_{1} d x_{2}
$$

coming from large $Q^{2}$ (see Eq. (2.8)):

$$
\ln \frac{\hat{s}}{\Lambda_{Q C D}^{2}}+\ln \left(4 \lambda_{0}\left(1-\lambda_{0}\right)\right) \gg 1
$$

$\delta q^{\gamma}$ is given by (4.1) and $q_{V}^{N} \approx x^{-\beta}$ for $x \rightarrow 0$ with $0<\beta<1$. Firstly, the contributions from arbitrarily large, but finite $\hat{s}$ region,

$$
\hat{s}_{1} \leq \hat{s} \leq \hat{s}_{2},
$$

$\hat{s}_{1}, \hat{s}_{2}$ independent of $s$, yield at most constant value. Next, take arbitrarily large, fixed $\hat{s}_{1}$ and

$$
\begin{gathered}
\hat{s}_{2} \simeq s^{\alpha}, \\
0<\alpha<1 .
\end{gathered}
$$


Parton cross sections in this region are

$$
\frac{d \sigma_{\text {part }}}{d \hat{t}}=\frac{f(\lambda)}{\hat{s}^{2}}
$$

with $M_{W}^{2}, M_{Z}^{2}$ neglected.

Integration in the subregion $\tau>1$ yields constant cross section only. The $\ln (s)$ contribution comes from saturation region, $\tau<1$, i.e.

$$
\begin{aligned}
& x_{1}^{-\Delta}>\frac{Q^{2}}{\bar{Q}_{0}^{2}}, \\
& \bar{Q}_{0}^{2}=Q_{0}^{2} x_{0}^{\Delta} .
\end{aligned}
$$

To simplify estimates I considered smaller integration region over $x$, i.e.

$$
x_{1}^{-\Delta}>\frac{\hat{s}}{\bar{Q}_{0}^{2}}
$$

and

$$
x_{2}<\frac{1}{A} .
$$

Then , taking $y=-\ln x$ the region of integration becomes

$$
\begin{aligned}
& \ln \frac{s}{\hat{s}_{2}}<y_{1}+y_{2}<\ln \frac{s}{\hat{s}_{1}}, \\
& (1+\Delta) y_{1}+y_{2} \geq \ln \frac{s}{\bar{Q}_{0}^{2}},
\end{aligned}
$$

and

$$
y_{2} \geq \ln A
$$

with $\alpha$ satisfying $\frac{\Delta}{1+\Delta} \leq \alpha \leq 1$ we get

$$
\Delta \sigma^{t o t} \approx \frac{c o n s t}{1-\beta}\left(\frac{1}{A}\right)^{1-\beta} \frac{\Delta}{1+\Delta} \ln \frac{s}{A \bar{Q}_{0}^{2}} .
$$

\section{Conclusions}

If, as we assumed, $\delta q^{\gamma}(x) / x$ does not vanish in the limit $x \rightarrow 0$ and scaling with saturation works, then for $s \rightarrow \infty$ the contribution of jets with $p^{T}$ larger than an arbitrarily large fixed value to the difference of the total cross sections $\Delta \sigma^{t o t}$ increases as $\ln (s)$, i.e. they contribute to maximal Odderon asymptotics.

\section{References}

[1] L. Łukaszuk, B. Nicolescu, Nuovo Cimento Lett. 8 (1973) 405.

[2] J. Kwiecinski, M. Praszalowicz, Phys. Lett. B94 (1980) 413.

[3] J. Bartels, L.N. Lipatov, G.P. Vacca, Phys. Lett. B477 (2000) 178. 
[4] C. Ewerz, arXiv:hep-ph/0306137.

[5] R. Avila, P. Gauron, B. Nicolescu, arXiv:hep-ph/0607089.

[6] L. Łukaszuk, Nucl. Phys. A709 (2002) 289.

[7] L.V. Gribov, E.M. Levin, M.G. Ryskin, Phys. Rept. 100 (1983) 1.

[8] I. Balitsky Nucl. Phys. B462 (1996) 99.

[9] Yu.V. Kovchegov, Phys. Rev. D60 (1999) 034008.

[10] A.M. Stasto, K. Golec-Biernat, J. Kwiecinski, Phys. Rev. Lett. 86 (2001) 596.

[11] J.L. Albacete et al., Phys. Rev. D71 (2005) 014003.

[12] J. Bartels, B.I. Ermolaev, M.G. Ryskin, Z. Phys. C70 (1996) 273.

[13] B.I. Ermolaev, M. Greco, S.I. Troyan, Phys. Lett. B579 (2004) 321.

[14] E. Iancu, K. Itakura, L. McLerran, Nucl. Phys. A708 (2002) 327.

[15] A.H. Mueller, D.N. Triantafyllopoulos, Nucl. Phys. B640 (2002) 331. 